\title{
Pharmacogenomics of cetuximab in metastatic colorectal carcinoma
}

Cetuximab is a chimeric monoclonal antibody that has revolutionized the treatment of metastatic colorectal cancer. Knowledge of the mechanisms that underlie its effectiveness, as well as the primary and secondary resistance mechanisms, have led to important developments in the understanding of cetuximab biology. In light of knowledge gained from recent trials, the efficacy of cetuximab has been clearly demonstrated to depend upon RAS mutational status, moreover cetuximab should only be used in a subset of patients who may benefit. In this article, we critically review clinical and pharmacogenetic issues of cetuximab, focusing on the cost-effectiveness involved with the use of the drug.

Keywords: cetxuximab • colorectal carcinoma $\bullet$ cost-effectiveness $\bullet$ EGFR

- pharmacogenomics $\bullet$ predictive $\bullet$ RAS $\bullet$ resistance

\section{Background}

Colorectal carcinoma (CRC) is the third most common cause of death in western countries [1]. Approximately a quarter of newly diagnosed patients have already developed metastases and almost $50 \%$ of all CRC patients will develop metastases over time. In the last decade, by best combining the three active cytotoxics available (fluoropyrimidines, irinotecan and oxaliplatin), median overall survival (mOS) of metastatic CRC (mCRC) patients has doubled from 10-12 months to more than 20 months [2]. More recently, the understanding of the biology of this malignancy has pointed towards potential targets for novel therapies. One of the most promising targets is the EGFR signaling pathway as a critical mechanism in CRC tumorigenesis [3].

Cetuximab is a chimeric IgG1 monoclonal antibody $(\mathrm{mAb})$ that competitively binds to the extracellular domain of EGFR, thus leading to inhibition of the downstream signaling pathways. This drug has been approved for mCRC therapy based on demonstrated improvement in overall survival (OS) when used either as a single agent or in combina- tion with chemotherapy [4]. Nevertheless, this drug is active only in a fraction of patients.

Nowadays, several studies provide evidence that a pharmacogenomics approach plays a key role in the identification of specific biomarkers that could identify patients who are most likely to respond to or be resistant to this costly $\mathrm{mAb}$. In particular, biomarker development for cetuximab has focused on the impact of alterations in EGFR and its downstream effectors with particular regards to KRAS, which encodes a small G-protein that functions downstream of EGFR-induced cell signaling [5]. However, although $K R A S$ mutations can be considered a highly specific negative biomarker for response to cetuximab, their absence does not mean the drug will be effective. This has led to the investigation of additional markers of primary resistance, thus focusing on the analysis of multiple genetic and epigenetic events involved downstream of EGFR signaling [6]. Recently, two large studies have shown that patients with mCRC harboring a mutation in KRAS or $N R A S$ did not have a response to anti-EGFR therapy, but for wild-type $K R A S$ and $N R A S$
Nicola Silvestris', Bruno Vincenzi ${ }^{2}$, Anna Elisabetta Brunetti', Fotious Loupakis ${ }^{3}$, Emanuela Dell'Aquila 2,3, Antonio Russo ${ }^{4}$, Mario Scartozzi ${ }^{5}$, Riccardo Giampieri ${ }^{5}$, Stefano Cascinu ${ }^{5}$, Vito Lorusso', Giuseppe Tonini $^{2}$, Alfredo Falcone ${ }^{3}$ \& Daniele Santini $i^{*}, 2$

${ }^{1}$ Medical Oncology Unit, National Cancer Institute "Giovanni Paolo II", Bari, Italy

${ }^{2}$ Department of Medical Oncology, University Campus Bio-Medico, Via Alvaro del Portillo, 200, 00128 Rome, Italy

${ }^{3}$ Oncology Center, Azienda OspedalieroUniversitaria Pisana, Istituto Toscano Tumori, Pisa, Italy

${ }^{4}$ Section of Medical Oncology, Department of Surgical, Oncological \& Stomatological Sciences, University of Palermo, Palermo, Italy

${ }^{5}$ Department of Medical Oncology, Translational Oncology Unit, AOU Ospedali Riuniti, Università Politecnica delle Marche, Ancona, Italy *Author for correspondence:

Tel.: +39062 25411344

Fax: +39062 25411206

d.santini@unicampus.it 
patients the addition of anti-EGFR $\mathrm{mAb}$ to first-line chemotherapy allowed for a 6-month OS advantage $[7,8]$. Indeed, research on the mechanisms of secondary acquired resistance is useful to define additional signaling pathways. Last but not least, the use of biomarkers to identify responsive/resistant patients has significant cost implications [9].

The objective of this review is to provide an update on the most recent data on mechanisms of primary and secondary resistance to cetuximab. Hence, pharmacogenetic testing issues, clinical results in mCRC patients and cost-effective analyses are discussed.

\section{Mechanism of action of cetuximab}

The HER family of receptors includes four distinct cell membrane-bound glycoproteins. EGFR is also known as HER1. The others are ERBB2, ERBB3 and ERBB4 $[10,11]$. Each of these receptors are structured into three regions: an extracellular ligand-binding region, a region which goes across cell membrane anchoring this receptor to the cell and an intracellular region with tyrosine kinase activity.

The extracellular domain of EGFR can bind six EGF-like ligands, with subsequent dimerization with other receptors of the HER family [12]. The dimerization leads to the activation of EGFR's tyrosine kinase activity so that the tyrosine residues in the intracellular domain are autophosphorylated. This intracellular activation of EGFR induces phosphorylation of other intracellular molecules, including those of RAS-RAF-MAPK cascade and PI3K-AKT-mTOR pathway.

RAS protein is active when it is bound to GTP, leading to subsequent activation of RAF protein. This molecule is a serine-threonine kinase and activates a cascade of proteins with the same kind of activity. This signaling is blocked when the GTP bound to RAS is hydrolyzed to GDP, the resulting RAS-GDP complex is inactive. As a consequence of the activation of this cascade even MAPK is active and it can translocate to the nucleus. The active MAPK in the nucleus regulates the function of several transcription factors. Subsequently multiple genes can change their own expression to regulate numerous cell functions including proliferation, angiogenesis, apoptosis, adhesion and motility [13,14].

Cetuximab binds to the extracellular domain of EGFR. This binding prevents EGFR from binding with endogenous ligands. This event blocks the receptor-dependent transduction pathway activation and its subsequent antitumor effects, including cell cycle arrest, induction of apoptosis, inhibition of angiogenesis and inhibition of metastasis. After the binding of EGFR with cetuximab this complex could undergo internalization and subsequent EGFR downregulation. Some relevant consequences also include enhancement of sensitivity to chemotherapy and radiation [15].

Cetuximab also showed cytotoxicity against tumor cells by antibody-dependent cellular cytotoxicity (ADCC). This phenomenon is mediated by the antibody Fc fragment, which can bind Fc $\gamma \mathrm{R}$ expressed on the cell surface of various immune cells, such as natural killer cells, monocytes or macrophages [16]. Even though $\mathrm{mAbs}$ are used in cancer treatment to block specific oncogenes, ADCC may be a phenomenon to explain their antitumor effects. Cetuximab has been included among drugs that could induce ADCC [17].

\section{EGFR pathway: mechanisms of primary \& secondary resistance \\ KRAS/BRAF pathway}

The rate of KRAS mutation is approximately $30-40 \%$ in mCRC [18]. KRAS mutations are point mutations mainly involving codon 12 and 13. These mutations represent up to $90 \%$ of all activating mutations in this gene. Other less frequent activating mutations include those in exon 2, codons 12 and 13; exon 3, codons 59 and 61; and exon 4, codons 117 and 146. G to A transitions and $\mathrm{G}$ to $\mathrm{T}$ transversions are observed most frequently. The most frequent $K R A S$ mutations, which are found in codons 12 and 13, cause impairment of the KRAS catalytic domain. The various changes in amino acids in the catalytic domain could induce differences in the intrinsic impairment of GTPase activity.

$R A S$ mutations may lead to different effects on cancer biology. Glycine to valine mutations in codon 12 have been associated with a more aggressive mCRC $[19,20]$. As a consequence of $R A S$ activating mutations, the RAS proteins become constitutively activated because of the stable RAS-GTP complex, which is not hydrolyzed. In fact the GTPase domain loses its intrinsic activity and becomes resistant to GTPase activating proteins. So mutated RAS proteins prompt signaling activation continuously, even though the upstream stimulations of EGFR is absent or blocked by anti-EGFR mAbs.

Usually KRAS mutations arise early in colon carcinogenesis, as supported by the evidence that KRAS mutation status in the samples of CRC primary tumor and metastases are highly concordant [21]. As some clinical studies showed, KRAS mutations have a relevant prognostic role in CRC patients, since these somatic gene mutations are more frequent in more advanced tumors and may relate to shorter survival. However the prognostic role of KRAS mutations is still controversial and more specific studies are necessary to strengthen this evidence [22-24].

Several retrospective analyses with a limited number of patients highlighted that KRAS mutation status 
negatively affects the antitumor activity of cetuximab, both as a single agent or in combination with chemotherapy. As a consequence just those patients with wild-type KRAS will respond to anti-EGFR-based treatment. These studies were performed in pretreated patients first. When a KRAS mutation was present the response rate (RR) was close to 0 . Subsequently even those studies for first-line treatment were re-evaluated for KRAS mutation status [25-30].

The incidence of $B R A F$ somatic mutations in CRC is approximately $15 \%$ [31]. These mutations are mutually exclusive in regards to $R A S$ mutations. Most frequently valine is substituted to glutamic acid (V600E) at position 600 of the RAF kinase domain. As a result a negatively charged residue is inserted at T598, which is adjacent to a site of regulatory phosphorylation. This event may mimic a regulatory phosphorylation. Kinase activity in a RAF mutated protein is elevated so that it is constitutively activated regardless of $R A S$ function. The continuous RAF activation prompts downstream MEK/ERK signaling, which is responsible for cell survival and proliferation. A half of all BRAF mutations are point mutations, even though other activating mutations promote signaling by an increased BRAF kinase activity or by indirect CRAF activation. Other mutations involve conserved amino acids next to the activation segment or glycine-rich loop [32,33] (Figure 1).

Other biomarkers have been studied to identify patients who can benefit from anti-EGFR therapy. Khambata-Ford $e t a l$. showed that patients with tumors that express high levels of epiregulin (EREG) and amphiregulin (AREG) are more likely to have disease control with cetuximab (EREG, $p=0.000015$; AREG, $\mathrm{p}=0.000025)$ and have significantly longer PFS than patients with low expression (EREG, $\mathrm{p}=0.0002$, hazard ratio [HR]: 0.47 and median PFS: 103.5 vs 57 days, respectively; AREG, $\mathrm{p}<0.0001$, HR: 0.44 and median PFS: 115.5 vs 57 days, respectively) [28].

Manceau et al., in a recent study, showed that a miRNA, has-miR-31-3p, could be a new marker for predicting survival in patients with wild-type KRAS mCRC treated with mAbs [34]. However, we do not have clear data about this yet.

\section{PTEN-PI3K-AKT-mTOR pathway}

PI3Ks are a family of lipid kinases that phosphorylate the 3 '-hydroxyl group of phosphoinositides with the conversion of phosphatidylinositol-4,5-biphosphate (PIP2) to phosphatidylinositol-3,4,5-trisphosphate (PIP3). The phosphate at position 3 of the inositol moiety of PIP3 is like a second messenger. AKT (also known as PKB) is activated by PDK1 through phosphorylation after the binding of PDK1 to PIP3. Once activated, phospho-
AKT phosphorylates up to 100 other proteins. mTOR is indirectly activated by AKT. This effect is mediated by the TSC complex. mTOR complexes with RAPTOR and RICTOR to form mTORC1 and mTORC2, respectively [6,35]. The activating mutations in PIK3CA, the gene encoding the p110 $\alpha$-catalytic subunit of PI3K, were identified as novel mechanisms of carcinogenesis. Somatic mutations of PIK3CA are present in over 25\% of colorectal and other malignancies. More than $80 \%$ of PIK3CA mutations in CRC occur in exon 9 (60-65\%) or exon $20(20-25 \%)$. These mutations can be concomitant with KRAS or BRAF mutations [36,37]. PIK3CA mutation in exon 9 is located in the helical domain of the PI3K protein. It induces a gain of function, which is independent of binding to the p85 regulatory subunit, but requires interaction with RAS-GTP. Conversely, exon 20 mutation is located in the kinase domain and is active in the absence of RAS-GTP binding, but is highly dependent on the interaction with $\mathrm{p} 85$. Some authors argue that the PIK3CA mutations in these two different sites imply opposite effects on the efficacy of anti-EGFR treatments [38].

PTEN is a phosphatase that antagonizes the PI3K/AKT signaling pathway by dephosphorylating PIP3 to inhibit activation of AKT with hyperactivation of PI3K signaling. PTEN seems to protect the genome from instability. The PTEN gene is activated in cancer cells by a combination of different molecular mechanisms (inactivating mutations, allelic losses and hypermethylation of the enhancer region) [39,40]. The loss of PTEN expression, as measured by immunohistochemistry, seems to be related to primary resistance to anti-EGFR mAbs in CRC. This loss of PTENexpression is concomitant with KRAS, BRAF and PIK3CA mutations, and EGFRamplification. The loss of PTEN expression ranges from 19 to $36 \%$. The loss of PTEN expression is usually not concordant in primary tumor and metastases. However the definition and evaluation of PTEN loss has not yet been standardized, so that PTEN expression cannot be a reliable biomarker of resistance to cetuximab in CRC [41,42] (Figure 1).

Recently, Martin et al. evaluated the ERBB2 gene status by FISH in 170 KRAS wild-type mCRC patients treated with cetuximab or panitumumab [41]. They concluded that a major ERBB2 gene amplification significantly correlated with worse clinical behaviors, in terms of response rate (RR), progression-free survival (PFS) and OS. This mechanism of primary resistance probably stems from the differences in ERBB2 gene deregulation. In particular, patients with $E R B B 2$ gene amplification may be resistant because "the majority of their cancer cells have the intrinsic ability to continuously activate downstream pathways", thereby overcoming the blockade of EGFR receptor [43]. These 


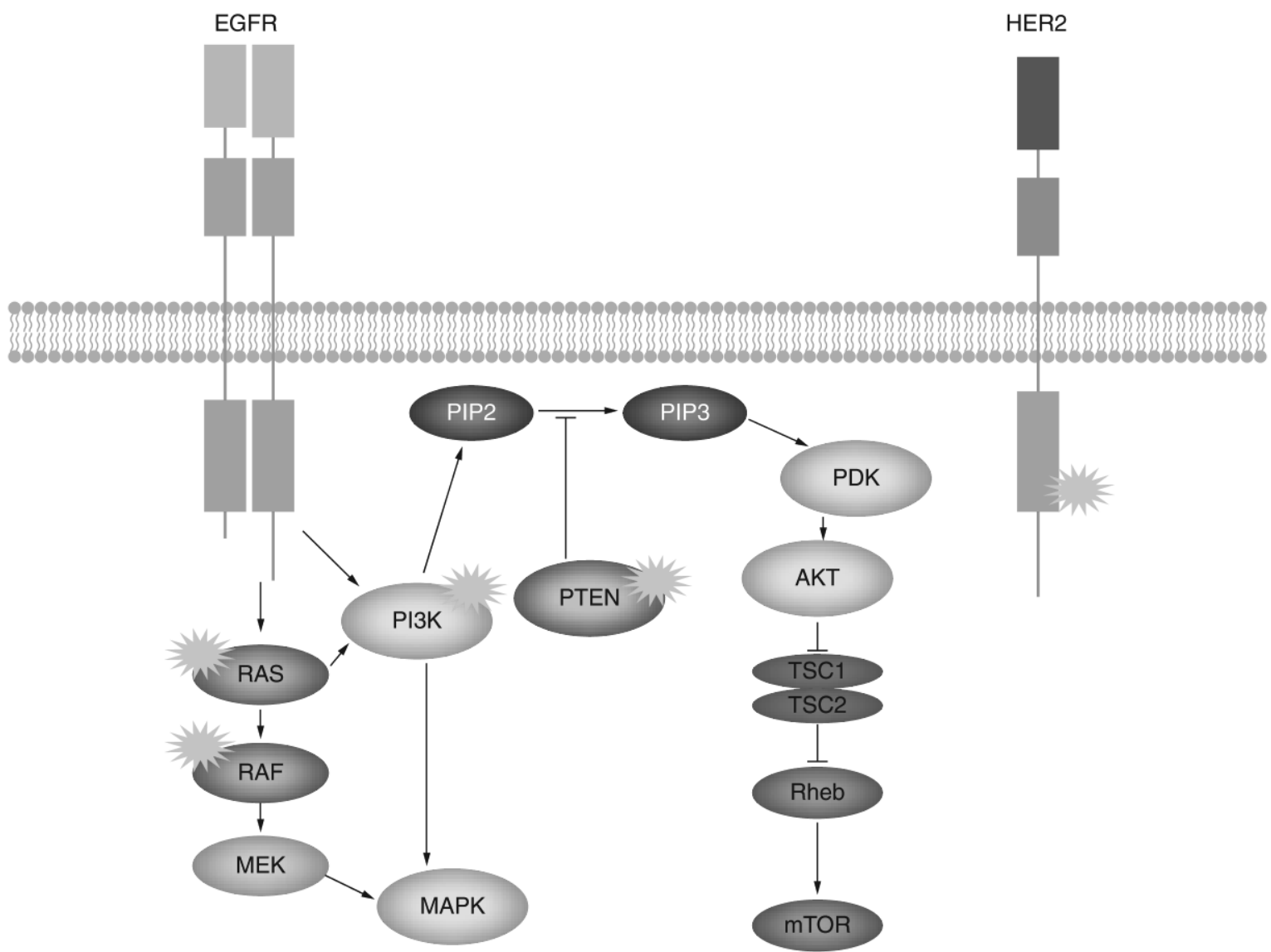

Figure 1. Genetic alterations predicting primary resistance to cetuximab.

results lay the groundwork for a therapy based on the simultaneous blocking of both ERBB2 and EGFR.

\section{Secondary resistance}

Patients with mCRC who respond to anti-EGFR mAbs ultimately acquire a secondary resistance to these agents. Comprehension of these additional resistance mechanisms is rarely understood, even if it is of clinical relevance for a better selection of the subset of mCRC patients likely to benefit from cetuximab or cetuximab-based combination therapies. Among the mechanisms of resistance, the oncogenic shift, constitutive activation of downstream pathways and increased angiogenesis were to be counted [44-46] (Figure 2). In particular, EGFR signaling can contribute to the release of several proangiogenic factors in the tumor microenvironment, including VEGF. VilorianPetit et al. have investigated VEGF-mediated secondary resistance in cell variants obtained from recurrent tumor xenografts of the human A431 squamous cell carcinoma [45]. The authors have shown that cell lines resistant to anti-EGFR mAbs were characterized by a more marked angiogenesis, after an initial suppression of the angiogenesis. Subsequently, Ciardiello et al. have confirmed that the anti-EGFR mAbs secondary resistance due to an increase of angiogenesis was overcome with the use of a dual inhibitor, ZD6474, a EGFR/VEGFR2 tyrosine kinase inhibitor, assuming a more effective strategy in resistant patients [46].

One of the theories for the onset of secondary resistance to EGFR blockade is represented by the presence of rare cells with KRAS mutations in tumors initially classified as KRAS wild-type. In fact, Diaz et al. described KRAS mutations in the circulating DNA of mCRC patients between 5 and 6 months following the initiation of a systemic therapy with anti-EGFR $\mathrm{mAb}[47]$. In an interesting work, some authors analyzed the behavior of two CRC cell lines (wild-type for KRAS, HRAS, NRAS, BRAF and PISKCA) incubated with cetuximab [48]. After repeated administration of the drug, one of the two cell lines became resistant to cetuximab through both the reduction of EGFR protein and the amplification of the KRAS protein. Sequence analysis showed that EGFR, KRAS, HRAS, NRAS, BRAF and PI3KCA genes remained wild-type in these clones. The second cell line developed resistance to cetuximab through the acquisition of either G13D or G12R KRAS mutations. Curiously, they 
observed the pre-existence of a small percentage of cells with these genetic alterations, but also the de novo acquisition of a KRAS mutation under the pressure of cetuximab treatment. These data are also supported by DNA analysis on metastases of patients treated with the mAb. In fact, some CRC patients, who exhibit an initial response to anti-EGFR therapies, have, at the time of disease progression, tumors with focal amplification or somatic mutations in KRAS, which were not detectable prior to initiation of therapy. Therefore, the authors concluded that drug resistance resulting from alterations in KRAS can be attributed not only to the selection of pre-existent KRAS mutant and amplified clones but also to new mutations that arise as the result of ongoing mutagenesis [48]. Remarkably, analysis of plasma samples from patients treated with cetuximab confirmed that the same $K R A S$ variants were present in post-treatment biopsies as early as 10 months before disease progression. Subsequently, the same authors demonstrated that, regardless of the genetic alterations, resistant cells consistently displayed MEK and ERK activation, which persisted after EGFR blockade, and the inhibition of MEK1/2 alone was not able to stop the growth of resistant cells in vitro and in vivo [49].
Instead, simultaneous inhibition of EGFR and MEK leads to a recovery of the secondary resistance in both in vitro experiments and in vivo, as shown by mouse xenograft experiments. Moreover, Bardelli and colleagues showed that the amplification of the MET proto-oncogene is associated with acquired resistance in tumors that do not develop $K R A S$ mutations during anti-EGFR therapy. This amplification was found by analysis of circulating DNA before the onset of clinical and radiological relapse/progression. Interestingly, in patient-derived colorectal cancer xenografts, the use of MET kinase inhibitors was able to overcome resistance to EGFR blockade [50].

Conversely, the tumor cell may amplify a dysregulated receptor. Wheeler et al. developed cells with acquired resistance to cetuximab in vitro by prolonging and escalating dose exposure to $\mathrm{mAb}$ [51]. These cells were characterized by increased EGFR expression associated with its altered internalization or degradation and HER3 activation. On the contrary, Lu et al. obtained resistant cells to cetuximab exposing the parental sensitive cells to subeffective doses of drug [52]. These cells showed markedly lower protein levels of EGFR, an increased association of EGFR with $\mathrm{Cbl}$

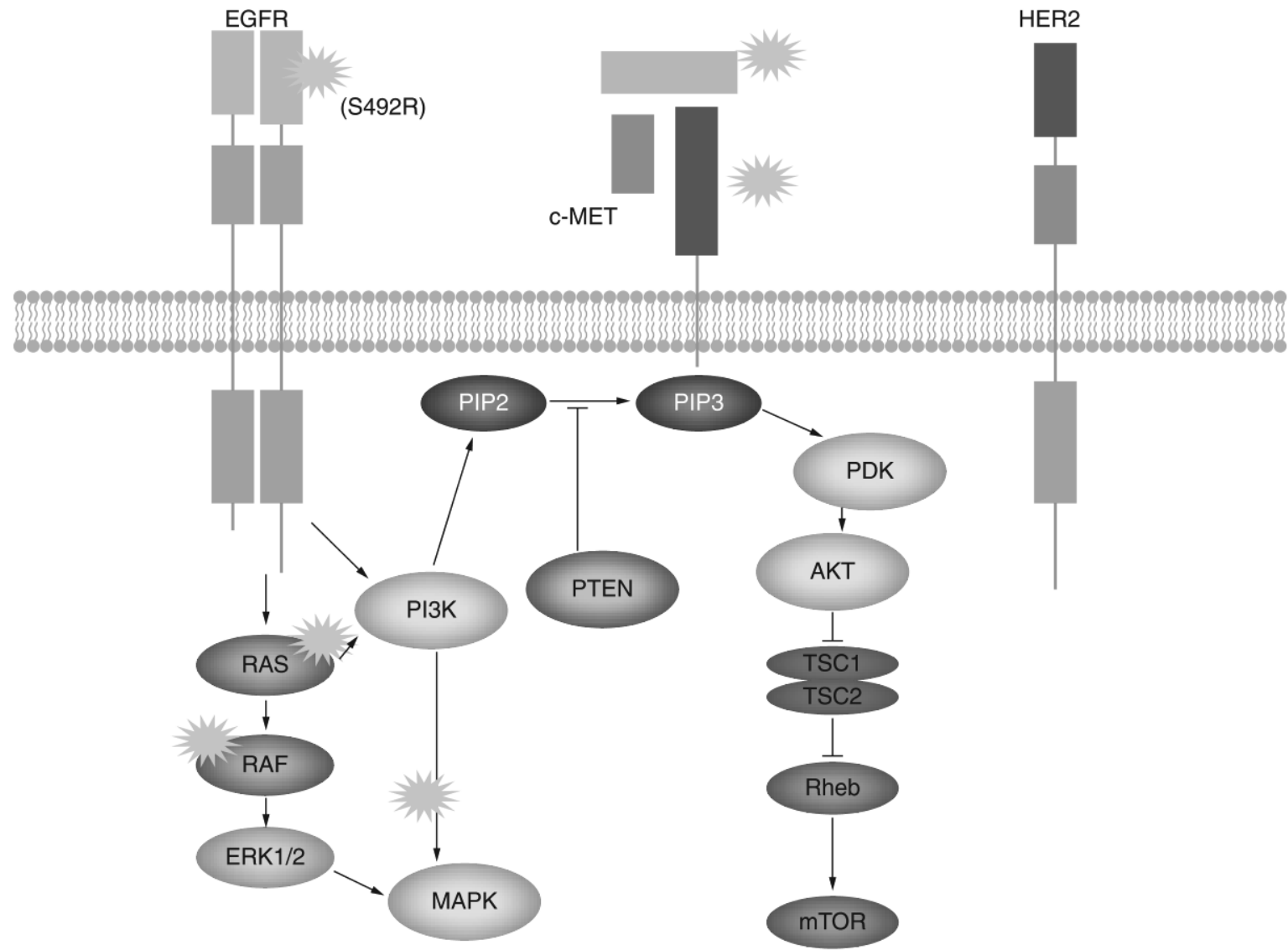

Figure 2. Genetic alterations predicting acquired resistance to cetuximab. 
protein and an increased ubiquitination of the receptor. Resistant cells also showed a markedly higher level of Src-Y416 phosphorylation at baseline and after EGF stimulation. This process is interrupted by inhibition of Src-Y416. Therefore, the authors concluded that EGFR ubiquitination and degradation promote the onset of an altered EGFR signal; however, using the inhibition of Src kinase-mediated cell signaling, it is possible to bypass the resistance.

Another possible mechanism of secondary resistance is represented by the appearance of mutations in the extracellular domain of EGFR. Montagut $e t a l$. observed that the S492R mutation conferred resistance to cetuximab maintaining susceptibility of tumor cells to panitumumab, supporting the hypothesis that this particular event may play a role in the development of secondary resistance to cetuximab [53] (Figure 2).

In a study by Tougeron et al., KRAS, BRAFV600E and EGFR S492R were analyzed simultaneously to evaluate the impact on treatment resistance in 23 mCRC consecutive patients retrospectively [54]. The results contrast with the data described above, as only a disease-control patient acquired a KRAS mutation during anti-EGFR $\mathrm{mAb}$ therapy, while this mutation was not found in any patient with progressive disease. In addition, by analyzing tissue samples from 37 patients treated with cetuximab, no EGFR S492R mutation was detected [54].

The secondary resistance to cetuximab could also be caused by the activation/amplification of a bypass signaling pathway. Yonesaka $e t$ al. uncovered aberrant HER2 signaling as a mediator of cetuximab resistance in a subset of cetuximab-resistant clones [55]. They also demonstrate that aberrant HER2 signaling contributes to both de novo and acquired drug resistance in cetuximab treated mCRC patients. Both mechanisms of aberrant HER2 activation lead to persistent ERK1/2 signaling in the presence of cetuximab, thus preventing cetuximab mediated growth inhibition. In addition to HER2 amplification, the increased levels of heregulin would lead to the association of the two receptors and their subsequent activation, leading to persistent activation of ERK signaling [55]. These results have been demonstrated by the authors in patients who became nonresponsive to cetuximab. In fact, higher levels of heregulin correlated with reduced PFS and OS, whereas the presence of HER 2 amplification correlated with worse OS.

The antitumor effect of cetuximab leads both to an increase in apoptosis and immune-mediated cytotoxicity. Lastly, recent data suggested the involvement of $\mathrm{T}$ cells in the antitumor activity of EGFR-targeted mAbs. Yang et al., through mouse tumor cell lines, demonstrated that cetuximab-induced tumor regres- sion depends on both innate and adaptive immunity components, such as $\mathrm{CD}^{+}{ }^{+} \mathrm{T}$ cells and Fc $\gamma \mathrm{R}$ [56]. Garrido et al. generated an in vivo model to study secondary resistance to EGFR-specific mAbs using immunocompetent mice, in order to demonstrate that, in addition to alterations in oncogenic signaling, defects in immunological pathways contribute to resistance induction in anti-EGFR mAb-treated tumors [57]. These results suggest that combinations of targeted therapies and immunomodulatory drugs may provide a successful strategy to overcome acquired resistance.

In conclusion, the understanding of the molecular mechanisms underlying the onset of secondary resistance may, in the future, allow for the identification of molecular targets against which to act.

\section{Liquid biopsy}

A new and very promising technique for the evaluation of secondary resistance consists of the evaluation of circulating tumor DNA in blood samples of patients treated with anticancer therapies. This approach is known as 'liquid biopsy'. This approach may be useful to easily achieve molecular information regarding metastatic disease, even before starting treatment with anti-EGFR agents, and to easily assess KRAS status. More specifically, the absence of detectable mutations in the primary tumor cannot formally exclude the presence of mutant metastatic diseases. Tumor heterogeneity and mutational selection during disease progression need further elucidation [58,59]. Recent studies have been performed to assess KRAS mutation status in circulating tumor DNA, with the view of improving patient selection. Lefebure et al. detected, in the serum of unresectable mCRC, KRAS mutation and RAS SF2A methylation and they observed that the presence of circulating mutant DNA was predictive of clinical outcome [60]. Bazan and colleagues demonstrated that the preoperative detection of mutant KRAS and TP53 in the serum of CRC patients undergoing resective surgery was predictive of disease recurrence [61]. However, the main purpose of KRAS analysis and DNA detecting in peripheral blood remains the monitoring of the secondary drug resistance acquisition. Two recent studies tried to explore the value of this new technique in this setting. As already mentioned above, in an in vitro model, Misale and colleagues demonstrated in eight out of 11 patients that cetuximab-resistant tumors had gained either KRAS mutation or amplification [48]. In addition, by analyzing plasma samples for circulating tumor DNA, the authors anticipated the identification of KRAS mutant alleles much before radiographic evidence of progression. The authors also demonstrated that the same $K R A S$ variants that were ultimately identified in the post-treatment (disease progression) biop- 
sies were detectable in plasma as early as 10 months prior to the documentation of disease progression by radiological assessment [48].

The approach used by Diaz et al. was more clinical and began with a cohort of KRAS wild-type mCRC patients treated with panitumumab [47]. The authors collected blood samples from each patient until the occurrence of disease progression. Among patients with initially $K R A S$ wild-type tumors, $38 \%$ developed detectable KRAS mutations during the course of therapy. This longitudinal collection of serum samples will allow clinicians to monitor the evolution of resistance over time, providing a tool that could anticipate the evidence of disease progression by standard radiologic and clinical criteria. Even if this amount of data offers a solid foundation for understanding acquired resistance to EGFR blockade in mCRC, much future work must be carried out in order to clarify and confirm the real impact on everyday practice in oncology. Moreover, while the current studies focused on DNA level changes - mutation and amplification - other mechanisms such as gene-expression changes and epigenetic alterations could be associated with the acquisition of resistance. Finally, issues about sensitivity and specificity of this assessment in order to correctly estimate the appearance of resistant clones must be the topic of future studies.

\section{Clinical studies in CRC}

Clinical studies exploring the role of cetuximab in different treatment lines have sometimes arrived at controversial results and raised doubts about the global impact of such a therapeutic option.

In the Phase III CRYSTAL trial [62], 1198 patients were randomized to receive FOLFIRI with or without cetuximab as the first-line chemotherapy option. The primary end point of the study was PFS, while OS and RR were secondary end points. In the primary analysis population, unstratified by KRAS status analysis, a significant improvement in PFS was seen (median PFS for FOLFIRI + cetuximab 8.9 months vs 8.0 months in the FOLFIRI group; hazard ratio [HR]: 0.85; 95\% CI: $0.72-0.99 ; \mathrm{p}=0.048$ ). There was no significant improvement in median OS (19.9 vs 18.6 months, respectively, for the FOLFIRI + cetuximab and FOLFIRI arms; HR: 0.93; 95\% CI: 0.81-1.07; $\mathrm{p}=0.31$ ). On the contrary, a significantly improved RR was seen in the FOLFIRI + cetuximab arm compared with the FOLFIRI arm (46.9 vs $38.7 \%$; odds ratio: 1.40 ; $\mathrm{p}=0.004)$.

At the time of study publication, $K R A S$ mutational status was available in 540 patients: $348(64 \%)$ were KRAS wild-type, whereas the remaining 192 (36\%) harbored either mutations in codon 12 or 13 . In the
$K R A S$ wild-type group, PFS as a result of the addition of cetuximab was significantly increased (median PFS, respectively, 9.9 vs 8.7 months; HR: $0.68,95 \%$ CI: $0.50-0.94 ; \mathrm{p}=0.02$ ) while OS still did not show a statistically significant improvement. An improved RR was also seen in the KRAS wild-type subgroup for the FOLFIRI + cetuximab arm versus the FOLFIRI arm (59.3 vs $43.2 \%$; odds ratio: 1.91 ; 95\% CI: $1.24-2.93$ ). In a subsequent publication [63], an updated survival analysis was presented, with the addition of an increased number (from 45 to $89 \%$ of the total population) of samples available for KRAS mutational status (codon 12 and 13). In this expanded population from the CRYSTAL trial, KRAS wild-type status was present in $666(62 \%)$ of the sampled population. Median PFS was still significantly improved in the KRAS wild-type population (9.9 vs 8.4 months; HR: 0.696; 95\% CI: $0.558-0.867 ; \mathrm{p}=0.0012$ ) and a significantly improved OS was seen for the $K R A S$ wild-type population (23.5 vs 20.0; HR: 0.796 ; $95 \%$ CI: $0.670-0.946$; $\mathrm{p}=0.0093)$. In the KRAS mutant subgroup, similar median PFS and median OS were seen. As expected RR was also improved in the KRAS wild-type population $(\mathrm{p}<0.001)$. In this expanded population BRAFV600E mutational analysis was also performed. Fifty-nine patients $(6 \%)$ harbored a V600E mutation of BRAF, 33 in the FOLFIRI + cetuximab arm and 26 in the FOLFIRI arm. Surprisingly no PFS or OS differences were evident for both treatments and even more surprisingly $B R A F$ mutant tumors showed a distinct profile in terms of prognosis. There was no evidence of an independent treatment by BRAF status interaction, and as a consequence authors were unable to definitely identify $B R A F$ mutational status as a predictor of resistance to cetuximab. On the contrary, a stronger prognostic role appeared more likely.

A subsequent analysis was conducted analyzing the impact of an extended RAS evaluation (KRAS exons 3 and 4 and NRAS exons 2, 3 and 4) on the patients outcome showing that patients with RAS wild-type tumors have a benefit from the addition of cetuximab to FOLFIRI.

Among 367 all-RAS wild-type patients, 178 were treated with FOLFIRI plus cetuximab, 189 with FOLFIRI alone: RR was 66.3 vs 38.6 ( $<<0.0001$ ), median progression-free survival 11.4 vs 8.4 ( $\mathrm{p}<0.0002)$, mOS 28.4 vs 20.2 ( $\mathrm{p}<0.0024)$ [64].

In the Phase III MRC COIN trial [65], 1630 patients were randomized to receive oxaliplatin + fluoropyrimidine based treatment with or without cetuximab. At the time of publication KRAS mutational status was available for 1316 (81\%) patients. OS was the primary end point of the study. Five hundred and sixty-five $(43 \%)$ patients harbored mutations in either 
codon 12 or 13 of the KRAS gene whereas the remaining 729 were wild-type. OS was not significantly different between the two treatment arms even in the KRAS wild-type population (17.9 vs 17.0 months; HR: 1.04; 95\% CI: 0.87-1.23; $\mathrm{p}=0.67$ ). No difference in PFS was seen in both the KRAS wild-type and KRAS mutant subgroup for the two treatment arms. The authors also conducted an analysis on BRAFV600E mutation and NRAS codon 12 and 13 mutations: 102 (8\%) patients harbored BRAF mutations whereas 50 (4\%) patients harbored an NRAS mutation. It must be noted that NRAS mutations were not mutually exclusive to both $B R A F$ and KRAS mutations. Analyzing the number of patients who did not harbor any mutation, the number of all-RAS wild-type patients dropped from $729(57 \%$ of the sampled population) to 581 (44\%). In this group of patients an improved median OS (20.1 months) was seen and it was significantly $(\mathrm{p}<0.0001)$ better than in patients harboring at least one mutation of either KRAS, NRAS or BRAF. Among patients harboring at least one mutation, those who had a BRAF mutation had a significantly worse OS than other groups (8.8 months). Owing to the results of this study the authors could not conclude that the addition of cetuximab to a first-line chemotherapy option yields a significant improvement in OS, even in a KRAS wild-type population.

In the Phase III NORDIC VII trial [66], 566 patients were randomly assigned to receive treatment with Nordic FLOX (FLOX: oxaliplatin $85 \mathrm{mg} / \mathrm{m}^{2}$ over

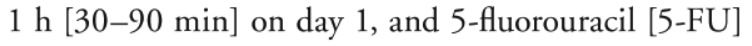
$500 \mathrm{mg} / \mathrm{m}^{2}$ as a bolus infusion $[<5 \mathrm{~min}]$, followed $30 \mathrm{~min}$ later by bolus folinic acid $60 \mathrm{mg} / \mathrm{m}^{2}[<10 \mathrm{~min}]$ on days 1 and 2, every 2 weeks) regimen \pm cetuximab continuously ( $\mathrm{arm} \mathrm{B})$ or intermittently $(\mathrm{arm} \mathrm{C})$. The authors also assessed the impact of KRAS codon 12 and 13 mutations ( 498 patients, $88 \%$ ) and the BRAF V600E mutation (457 patients, 81\%). KRAS mutations were present in $39 \%$ of the samples whereas $B R A F$ mutations were present in $12 \%$ of the samples. Primary end point of the study was PFS whereas RR and OS were secondary end points. No significant differences could be found in PFS in the primary population unstratified by KRAS status. Median PFS for arms $\mathrm{A}, \mathrm{B}$ and $\mathrm{C}$ were 7.9, 8.3 and 7.3 months, respectively. $\mathrm{HR}$ for comparison between arms $\mathrm{A}$ and B was not statistically significant. This was also observed in the $K R A S$ wild-type population. Paradoxically, in KRAS mutant patients, a not significant trend towards improved PFS was seen for patients receiving cetuximab (median PFS for arm A 7.8 months vs 9.2 months for arm B). No difference in PFS could be found for patients with $K R A S$ wild-type or mutant tumor regardless of treatment arm. OS was also not significantly different among the three treatment arms. Similar OS findings were evident for KRAS wild-type or mutant patients (median OS 21.0 vs 20.5 months, respectively; $\mathrm{p}=0.96$ ). The only subgroup performing differently was the $B R A F$ mutant group. In the 55 patients who harbored the BRAF V600E mutation, median PFS was 5.1 vs 8.3 months in the BRAF wild-type patients $(\mathrm{p}<0.001)$. A significantly shorter OS was also seen for $B R A F$ mutant patients vs wildtype (9.5 vs 22 months; $\mathrm{p}<0.001)$. In the FIRE-03 trial [67], patients were randomized to receive either FOLFIRI + cetuximab or FOLFIRI + bevacizumab as first-line treatment. Primary end point of the study was RR. Seven hundred and fifty-two patients were enrolled and among them 592 patients were KRAS wild-type (codon 12 and 13). In this population no significant difference in terms of overall response was noted. Median PFS was also not statistically different between the two treatment arms (10.0 vs 10.3 months; $\mathrm{p}=0.547)$. Perhaps the most striking result is that the study showed a significantly improved OS for patients receiving FOLFIRI + cetuximab vs FOLFIRI + bevacizumab (28.7 vs 25.0 months; $\mathrm{p}=0.017$ ). A subsequent analysis was conducted analyzing the impact of an extended $R A S$ evaluation on the patients outcome [8]. All-RAS mutational analysis was successfully conducted on 407 patients. Among them, 342 patients were all- $R A S$ wild-type whereas the remaining 65 harbored secondary mutations in either NRAS or KRAS gene 'hotspots', thus identifying another additional $16 \%$ of patients most likely to not benefit from antiEGFR treatment. Among the 342 all- $R A S$ wild-type patients, 171 were treated with FOLFIRI + cetuximab whereas the remaining 171 received FOLFIRI + bevacizumab. In this selected population, primary end point (RR) was still not significantly different, albeit a trend towards improved RR was seen in the FOLFIRI + cetuximab arm (65.6 vs $59.6 \% ; \mathrm{p}=0.32)$. PFS was also not significantly different between the two treatment arms even in this selected subgroup. The difference in OS that was observed in the KRAS limited analysis was heightened when assessing the impact of the extended RAS evaluation: patients with all-RAS wild-type status treated with FOLFIRI + cetuximab achieved a median OS of 33.1 months vs 25.6 months for the FOLFIRI + bevacizumab group (HR: 0.70; 95\% CI: 0.53-0.92; $\mathrm{p}=0.011$ ).

Although results from these trials may be difficult to interpret, a message about the critical role of an all- $R A S$ evaluation for first-line anti-EGFR therapy clearly emerged. The use of cetuximab was also explored in subsequent treatment lines. In the NCIC CTG/AGITG CO.17 trial [68], 572 patients who were initially treated with 5-FU, oxaliplatin and irinotecan 
and who had a positive immunohistochemistry EGFR tumor expression were randomized to receive cetuximab + best supportive care or best supportive care alone. Primary end point of the study was OS. The study met its primary end point, showing an improvement in OS in patients who received cetuximab (6.1 vs 4.6 months median OS time; $\mathrm{p}=0.005$ ). A significantly improved PFS was also seen for patients who received cetuximab (HR: 0.68; $\mathrm{p}=0.001$ ). After reanalysis of the trial according to KRAS mutational status (codon 12 and 13) a stronger effect for the use of cetuximab became evident [69]. Particularly, KRAS wild-type patients receiving cetuximab showed a statistically significant benefit in OS (9.5 vs 4.8 months; $\mathrm{p}<0.001$ ).

One interesting consideration that can be drawn from the results of these studies is that combinations where cetuximab is used with oxaliplatin, particularly in $R A S$ mutant patients, are generally met with failure, whereas a higher advantage from treatment can be observed in studies where cetuximab is given in monotherapy or in addition to irinotecan. The explanation is far from being understood: indeed, in the OPUS trial [70], a randomized Phase II trial of firstline treatment with FOLFOX $4 \pm$ cetuximab, while in KRAS patients a significantly improved RR, OS and PFS was observed for patients treated with the combination (mirroring the effect seen in the previous CRYSTAL trial), a significantly decreased RR (34 vs $53 \% ; \mathrm{p}=0.0290$ ), PFS (5.5 vs 8.6 months; HR: 1.720 ; 95\% CI: $1.104-2.679 ; \mathrm{p}=0.0153)$ and a trend for worse OS (13.4 vs 17.5 months; HR: 1.290 ; 95\% CI: $0.8703-1.906 ; \mathrm{p}=0.20$ ) was observed for patients with KRAS mutant status when treated with cetuximab vs control group. This negative effect is even more evident in trials where 5-FU is given orally such as in XELOX + cetuximab [65]. It is also an effect seen in patients treated with the other viable anti-EGFR monoclonal antibody in use, panitumumab. Indeed, despite the PRIME study showing a significantly improved survival for patients with all- $R A S$ wild-type status [7], in the RAS mutant population a significantly decreased survival can be seen when these subjects are treated with panitumumab + FOLFOX4.

While a conclusive answer to this question is still lacking, some preclinical data point out at the reasons of this possible interaction: it is hypothesized that in KRAS mutant cells there is a different EGFR localization compared with $K R A S$ wild-type cells (intracellular vs on cell surface expression, respectively). This may partly be reverted by use of drugs active on the intracellular portion of EGFR such as gefitinib [71]. In the absence of a clear answer to this question, caution must be advised when employing oxaliplatin + cetux- imab chemotherapy combinations, particularly in the first-line setting and especially when the 5-FU regimen used is based on oral capecitabine (Table 1).

\section{Pharmacogenetic testing issues}

Pharmacogenetic studies aim at identifying functional polymorphisms, which affect gene-expression regulation and can contribute to the differences between patients in terms of disease severity and treatment response [72]. This approach to the discovery of clinically useful biomarkers has several intrinsic advantages, such as ease of execution, reproducibility and handiness. Many data concerning SNPs related to prognosis and to the prediction of benefit from drugs employed in $\mathrm{mCRC}$ are available. Despite the large amount of available data, there are no SNPs routinely adopted in clinical practice, owing to the wide amount of contrasting results and the absence of confirmatory trials.

Many SNPs in genes from pathways more or less directly involved in anti-EGFRs mechanism of action have been identified. A germline polymorphism that consists of the variable number of the CA tandem repeats (rs45559542) at intron 1 of the EGFRgene has been extensively studied. In experimental models the transcription of the EGFR gene was found to be deeply inhibited in EGFRintron 1 alleles with a higher number of CA tandem repeats, whereas decreasing the number of CA pairs enhances transcription [73]. The first translational study in mCRC was conducted in a cohort of 39 patients treated with single-agent cetuximab in third-line therapy or after. An increasing number of repeats was associated with a low chance of objective response ( 0 vs $13 \% ; \mathrm{p}=0.94$ ) although the small number of patients and absence of $K R A S$ status data could explain the lack of significance [74]. In a larger study of 110 chemorefractory mCRC patients treated with cetuximab and irinotecan, a significant association with favorable PFS and OS was observed for EGFR intron 1 short (CA repeats $<17)$ genotype in a multivariate analysis (HR: $0.41, \mathrm{p}=0.006$; HR: $0.41, \mathrm{p}=0.006$, respectively) [75]. Conversely, the retrospective evaluation of EGFR intron 1 (both alleles with repeats $<20$ vs any allele with repeats $\geq 20$ ) in $130 \mathrm{mCRC}$ patients enrolled in the Phase II IMC-0144 clinical trial treated with single-agent cetuximab showed no significant association with clinical outcome [76]. More recently, the comparative analysis of a two arm trial in KRAS wild-type patients treated with chemotherapy and bevacizumab with or without cetuximab as first-line, showed that the EGFR intron 1 long variant (CA repeats $>20)$ was significantly related to a decreased PFS in the cetuximab arm (HR: $1.58 ; \mathrm{p}=0.024)$, while the EGFR (CA)n status did not influence the outcome of patients treated without cetuximab [77]. Major limi- 


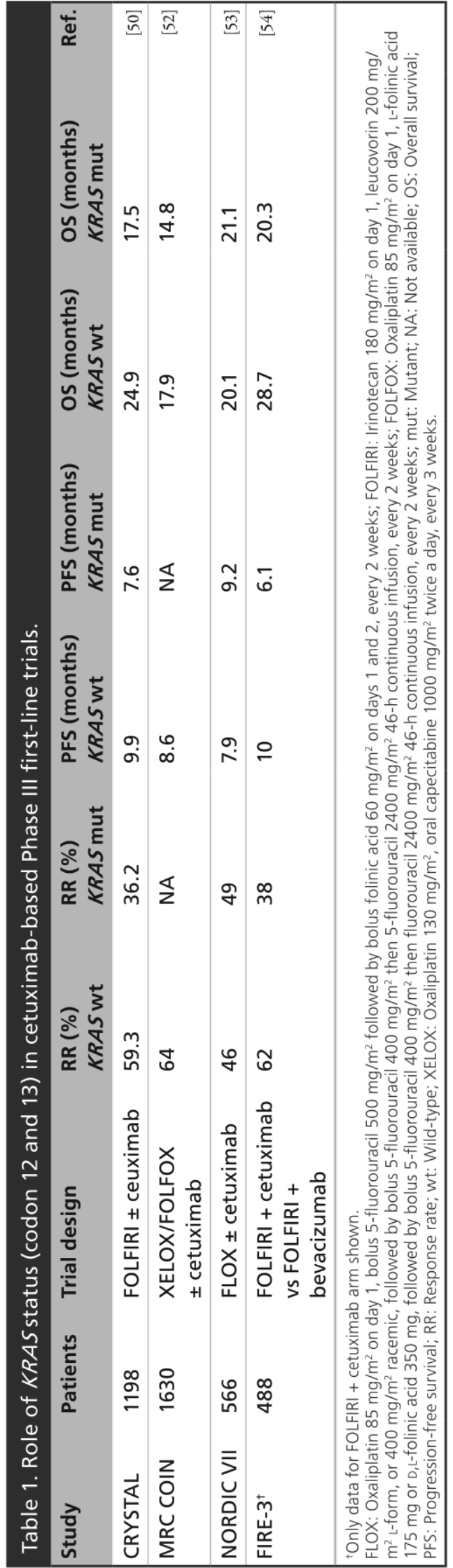

tations of the above mentioned studies were the different cutoff values for the number of CA repeats adopted and that $K R A S$ mutational status was not evaluated. In order to prospectively evaluate the role of EGFR intron 1 (CA)n repeat, an international collaborative group conducted a confirmatory study to detect a HR for PFS of 1.75 for L- compared with SS genotypes in a clinically and moleculary selected population. The study included a total of 113 KRAS and BRAF wildtype irinotecan-resistant mCRC patients treated with biweekly cetuximab and irinotecan in advanced lines. Short $(S)$ or long $(\mathrm{L})$ allelic variants were defined as those presenting $<20$ and $\geq 20$ CA repeats, respectively. No differences in PFS were observed between L- and SS genotypes (median progression-free survival: $4.4 \mathrm{vs}$ 5.3 months; HR: 1.00; $\mathrm{p}=0.991$ ); no differences in OS were observed as well (mOS: 11.3 vs 14.2 months; HR: $1.30 ; \mathrm{p}=0.261$ ). Other exploratory analyses adopting different cutoff values reported in the literature led to similar negative results [78]. Those results did not confirm any predictive or prognostic effect for $E G F R(\mathrm{CA}) \mathrm{n}$ repeat allelic variants with respect to the efficacy of cetuximab and irinotecan in advanced lines of treatment.

Other than directly blocking EGFR, cetuximab and panitumumab may recruit host effectors that determine an ADCC through the Fc $\gamma \mathrm{R}$ IIa and IIIa. In a preliminary study of 36 patients treated with singleagent cetuximab, Zhang $e t$ al. showed that the somatic alleles Fc $\gamma$ RIIa-131A (rs1801274) or Fc $\gamma$ RIIIa-158T (rs396991) were associated with a longer PFS (HR: 1.43, $\mathrm{p}=0.037$; and HR: 2.28, $\mathrm{p}=0.055$, respectively) [79]. In another experience conducted in 69 irinotecan-refractory $\mathrm{mCRC}$ patients treated with cetuximab plus irinotecan, subjects with Fc $\gamma$ RIIa- $131 \mathrm{H} / \mathrm{H}$ and/or Fc $\gamma$ RIIIa$158 \mathrm{~V} / \mathrm{V}$ genotypes had longer PFS than $131 \mathrm{R}$ and $158 \mathrm{~F}$ carriers (5.5 vs 3.0 months; $\mathrm{p}=0.005$ ) [80]. Two other larger experiences, one of 110 advanced line patients treated with cetuximab plus irinotecan, and another of 472 KRAS wild-type chemorefractory patients, did not confirm previously reported observations [75-81]. Again, the need for independent replication and prospective confirmation of exploratory pharmacogenetic studies is underlined.

Another promising field for the discovery of new biomarkers is that of miRNAs. miRNAs are a class of small RNAs that, exerting an effect as either tumor suppressors or oncogenes, may regulate several genes. Members of the Let-7 family of miRNAs showed RAS regulating activity and their possible role in influencing benefit from anti-EGFRs has been investigated. Two retrospective studies examined the association between the SNP on the Let-7 binding-site, named LCS6 (rs61764370), and cetuximab efficacy. In patients 
treated with cetuximab monotherapy after failure of previous regimens, KRAS wild-type patients harboring at least one $\mathrm{G}$ allele had a better outcome in terms of RR ( $p=0.02)$ compared with those with the TT genotype [82]. A retrospective analysis of $134 \mathrm{mCRC}$ irinotecan-refractory patients treated with cetuximab plus irinotecan suggested that LCS6 G allele may be associated with a shorter PFS (HR: 1.59; $\mathrm{p}=0.03$ ) and OS (HR: $1.68 ; \mathrm{p}=0.002$ ) [83].

Another attempt carried out in the field of pharmacogenetics was to correlate allelic variants of candidate SNPs to skin toxicity from EGFR inhibitors. In a recently published experience, Parmar et al. showed that EGFR 497G/A, the haplotypes of the promoter variants, EGFR-216G/T and -191C/A, and a haplotype in the PIK3CA gene could be associated with the occurence of cutaneous rash. Results need further evaluation before any possible clinical application [84].

\section{Cost-effectiveness analysis}

Although the clinical evidence has demonstrated benefits associated with cetuximab in mCRC, increasing its utilization influenced the already significant economic impact related to the treatment of this malignancy [85]. Thus, rising costs in this field cause discussions about the reimbursement of drugs as well as adequate costeffectiveness analyses [86]. The main aim of these evaluations is to compare differences in costs to differences in

\section{Executive summary}

\section{Mechanism of action of cetuximab}

- Cetuximab has been approved for metastatic colorectal carcinoma (mCRC) therapy based on demonstrated improvement in overall survival (OS) when used either as single agent or in combination with chemotherapy.

- Cetuximab binds to the extracellular domain of the EGFR, preventing the EGFR from binding with endogenous ligands. This event blocks the receptor-dependent transduction pathway activation with subsequent antitumor effects.

EGFR pathway: mechanisms of primary \& secondary resistance \& liquid biopsy

- KRAS mutation is the first established molecular marker that precludes responsiveness to EGFR-targeted treatment with cetuximab.

- Patients with $\mathrm{mCRC}$ harboring a mutation in KRAS or NRAS did not have a response to anti-EGFR therapy.

- The activating mutations in PIK3CA can be concomitant with KRAS or BRAF mutations and PIK3CA mutations in two different sites imply opposite effects in the efficacy of anti-EGFR treatments.

- PTEN is a phosphatase that antagonizes the PI3K/AKT signaling pathway; the loss of PTEN expression seems to be related to primary resistance to anti-EGFR monoclonal antibodies in colorectal carcinoma.

- Comprehension of secondary resistance mechanisms is of clinical relevance for a better selection of the subset of $\mathrm{mCRC}$ patients likely to benefit from cetuximab or cetuximab-based combination therapies.

- RAS-related drug resistance can develop as a consequence of both a natural selection and amplification of pre-existing clones bearing a somatic RAS mutation and an acquirement of new mutations that arise because of ongoing mutagenesis.

- Even if several data offer a solid foundation for understanding acquired resistance to EGFR blockade in $\mathrm{mCRC}$, much future work must be carried out in order to clarify and confirm the real impact on the everyday practice in oncology.

\section{Pharmacogenetic testing issues}

- Pharmacogenetic studies aim at identifying functional polymorphisms, which affect gene-expression regulation and can contribute to the differences between patients in terms of disease severity and treatment response.

- Many data concerning SNPs related to prognosis and to the prediction of benefit from drugs employed in $\mathrm{mCRC}$ are available.

- A germline polymorphism that consists of a variable number of CA tandem repeats (rs45559542) at intron 1 of the EGFR gene has not shown definite association with clinical outcome.

Clinical studies in colorectal carcinoma

- In the Phase III CRYSTAL trial a significantly improved response rate was seen in the FOLFIRI + cetuximab arm compared with the FOLFIRI arm. Median progression-free survival was still significantly improved in the $K R A S$ wild-type population and a significantly improved OS was seen for the KRAS wild-type population.

- In the FIRE-03 trial, patients with all-RAS wild-type status treated with FOLFIRI + cetuximab achieved a median OS of 33.1 months versus 25.6 months for the FOLFIRI + bevacizumab group.

- Although results from clinical trials may be difficult to interpret, a message about the critical role of an all-RAS evaluation for first-line anti-EGFR therapy clearly emerged.

Cost-effectiveness analysis

- The detection of RAS mutations prior to the administration of cetuximab was found to be clearly associated with cost savings in studies considering cetuximab treatment in first, second or subsequent treatment lines. 
health effects between alternative therapies to support the decision process. In this scenario, cost-effectiveness analyses of pharmacogenomics profiling appeared of paramount importance. In fact, although the implementation of KRAS testing is associated with additional upfront costs, as might be expected, overall, it is found to be clearly cost effective compared with no testing [87]. In particular, the detection of KRAS mutations prior to the administration of cetuximab was found to be clearly associated with cost savings in studies considering cetuximab treatment in first, second or subsequent treatment lines [88-92]. Using KRAS testing to select mCRC patients for EGFR-targeted $\mathrm{mAb}$ therapy saved US $\$ 7500-$ US $\$ 12,400$ per patient in the USA and $€ 3900-€ 9600$ per patient in Germany [90]. Indeed, in another European cost-effectiveness analysis, using $K R A S$ testing to limit treatment to patients with $K R A S$ wild-type tumors led to savings of $€ 779.42$ per patient per cycle [93].

\section{Conclusion}

Cetuximab has allowed for significant advantages in terms of RR, PFS and OS in a subset of $m C R C$ patients. However, the benefit form anti-EGFR based anticancer therapy can be largely variable among patients both in terms of entity and duration of response. In fact, it is becoming increasingly apparent that disease progression is largely driven by complex pathways and analysis of one single marker is unlikely to predict progression of disease with a high degree of accuracy and

\section{References}

Papers of special note have been highlighted as:

- of interest; $\bullet$ of considerable interest

1 Ferlay J, Shin HR, Bray F, Forman D, Mathers C, Parkin DM. Estimates of worldwide burden of cancer in 2008: GLOBOCAN 2008. Int. J. Cancer 127, 2893-2917 (2009).

2 Grothey A, Sargent D, Goldberg RM, Schmoll HJ. Survival of patients with advanced colorectal cancer improves with the availability of fluorouracil-leucovorin, irinotecan, and oxaliplatin in the course of treatment. J. Clin. Oncol. 22, 1209-1214 (2004).

3 Custodio A, Feliu J. Prognostic and predictive biomarkers for epidermal growth factor receptor-targeted therpy in colorectal cancer: beyond KRAS mutation. Crit. Rev. Oncol. Hematol. 85, 45-81 (2013).

4 Heinemann V, Douillard JY, Ducreux M, Peeters M. Targeted therapy in metastatic colorectal cancer - an example of personalized medicine in action. Cancer Treat. Rev. 39, 592-601 (2013).

5 Silvestris N, Tommasi S, Santini D et al. KRAS mutations and sensitivity to anti-EGFR monoclonal antibodies in metastatic colorectal carcinoma: an open issue. Expert Opin. Biol. Ther: 9, 565-577 (2009). reproducibility. To date, except for the predictive factors already known (KRAS and NRAS), there is some data that BRAF and PIK3CA activating mutations and PTEN inactivation confirm relative resistance to cetuximab. In addition, data concerning SNPs and miRNA related to prognosis and to the prediction of benefit from cetuximab employed in mCRC are available. In this scenario, the possibility of exploiting the liquid biopsy approach may allow future clinical trials to be initiated as soon as the resistant clones emerge.

\section{Future perspective}

Future studies should be performed to identify novel molecular targets and develop effective targeting strategies in this subset of patients. In particular, alterations in other key elements of the EGFR-dependent signal cascade (MAPK or AKT), or the presence of genetic alterations in receptor tyrosine kinases other than EGFR is also warranted to define additional signaling pathways to be targeted.

\section{Financial \& competing interests disclosure}

The authors have no relevant affiliations or financial involvement with any organization or entity with a financial interest in or financial conflict with the subject matter or materials discussed in the manuscript. This includes employment, consultancies, honoraria, stock ownership or options, expert testimony, grants or patents received or pending, or royalties.

No writing assistance was utilized in the production of this manuscript.
6 Silvestris N, Tommasi S, Petriella D et al. The dark side of the moon: the PI3K/PTEN/AKT pathway in colorectal carcinoma. Oncology77(Suppl. 1), 69-74 (2009).

7 Douillard JY, Oliner KS, Siena S et al. Panitumumab-FOLFOX 4 treatment and RAS mutations in colorectal cancer. N. Engl. J. Med. 369(11), 1023-1034 (2013).

8 Stintzing S, Jung A, Rossius L et al. Analysis of KRAS/NRAS and BRAF mutations in FIRE-3. Presented at: 17th ECCO, 38th ESMO and 32nd ESTRO European Cancer Congress (ECC 2013). Amsterdam, The Netherlands, 27 September-1 October 2013.

- In a subsequent analysis, patients with all- $R A S$ wild-type status treated with FOLFIRI + cetuximab achieved a median overall survival of 33.1 months versus 25.6 months for the FOLFIRI + bevacizumab group.

9 Davis JC, Fustenthal L, Desai AA et al. The microeconomics of personalized medicine: today's challenge and tomorrow's promise. Nat. Rev. Drug Discov. 8, 279-286 (2009).

10 Wong SF. Cetuximab: an epidermal growth factor receptor monoclonal antibody for the treatment of colorectal cancer. Clin. Ther: 27(6), 684-694 (2005).

11 Pérez-Soler R. HER1/EGFR targeting: refining the strategy. Oncologist 9, 58-67 (2004). 
12 Anido J, Matar P, Albanell J et al. ZD1839, a specific epidermal growth factor receptor (EGFR) tyrosine kinase inhibitor, induces the formation of inactive EGFR/HER2 and EGFR/ HER 3 heterodimers and prevents heregulin signaling in HER2-overexpressing breast cancer cells. Clin. Cancer Res. 9(4), 1274-1283 (2003).

13 Bronte G, Cicero G, Cusenza S et al. Monoclonal antibodies in gastrointestinal cancers. Expert Opin. Biol. Ther: 13(6), 889-900 (2013).

14 Caraglia M, Santini D, Bronte G et al. Predicting efficacy and toxicity in the era of targeted therapy: focus on anti-EGFR and anti-VEGF molecules. Curr. Drug Metab. 12(10), 944-955 (2011).

15 Martinelli E, De Palma R, Orditura M, De Vita F, Ciardiello F. Anti-epidermal growth factor receptor monoclonal antibodies in cancer therapy. Clin. Exp. Immunol. 158(1), 1-9 (2009).

16 Alderson KL, Sondel PM. Clinical cancer therapy by NK cells via antibody dependent cell-mediated cytotoxicity. J. Biomed. Biotechnol. 2011, 379123 (2011).

17 Weiner LM, Surana R, Wang S. Monoclonal antibodies: versatile platforms for cancer immunotherapy. Nat. Rev. Immunol. 10(5), 317-327 (2010).

18 Andreyev HJ, Norman AR, Cunningham D et al. Kirsten ras mutations in patients with colorectal cancer: the 'RASCAL II' study. Br. J. Cancer 85(5), 692-696 (2001).

19 Boughdady IS, Kinsella AR, Haboubi NY, Schofield PF. K-ras gene mutations in adenomas and carcinomas of the colon. Surg. Oncol. 1(4), 275-282 (1992).

20 Heinemann V, Stintzing S, Kirchner T, Boeck S, Jung A. Clinical relevance of EGFR- and KRAS-status in colorectal cancer patients treated with monoclonal antibodies directed against the EGFR. Cancer Treat. Rev. 35(3), 262-271 (2009).

21 Santini D, Loupakis F, Vincenzi B et al. High concordance of $K R A S$ status between primary colorectal tumors and related metastatic sites: implications for clinical practice. Oncologist 13(12), 1270-1275 (2008).

22 Bazan V, Migliavacca M, Zanna I et al. Specific codon 13 K-ras mutations are predictive of clinical outcome in colorectal cancer patients, whereas codon $12 \mathrm{~K}$-ras mutations are associated with mucinous histotype. Ann. Oncol. 13(9), 1438-1446 (2002).

23 Samowitz WS, Curtin K, Schaffer D, Robertson M, Leppert M, Slattery ML. Relationship of Ki-ras mutations in colon cancers to tumor location, stage, and survival: a populationbased study. Cancer Epidemiol. Biomarkers Prev. 9(11), 1193-1197 (2000).

24 Russo A, Rizzo S, Bronte G et al. The long and winding road to useful predictive factors for anti-EGFR therapy in metastatic colorectal carcinoma: the KRAS/BRAF pathway. Oncology 77(Suppl. 1), 57-68 (2009).

25 Lièvre $\mathrm{A}$, Bachet $\mathrm{JB}$, Boige $\mathrm{V}$ et al. KRAS mutations as an independent prognostic factor in patients with advanced colorectal cancer treated with cetuximab. J. Clin. Oncol. 26(3), 374-379 (2008).

26 De Roock W, Piessevaux H, De Schutter J et al. KRAS wildtype state predicts survival and is associated to early radiological response in metastatic colorectal cancer treated with cetuximab. Ann. Oncol. 19(3), 508-515 (2008).
27 Di Fiore F, Blanchard F, Charbonnier F et al. Clinical relevance of KRAS mutation detection in metastatic colorectal cancer treated by cetuximab plus chemotherapy. Br. J. Cancer 96(8), 1166-1169 (2007).

28 Khambata-Ford S, Garrett CR, Meropol NJ et al. Expression of epiregulin and amphiregulin and $K$-ras mutation status predict disease control in metastatic colorectal cancer patients treated with cetuximab. J. Clin. Oncol. 25(22), 3230-3237 (2007).

29 Benvenuti S, Sartore-Bianchi A, Di Nicolantonio F et al. Oncogenic activation of the RAS/RAF signaling pathway impairs the response of metastatic colorectal cancers to antiepidermal growth factor receptor antibody therapies. Cancer Res. 67(6), 2643-2648 (2007).

Amado R, Wolf M, Peeters M et al. Wild-type KRAS is required for panitumumab efficacy in patients with metastatic colorectal cancer J. Clin. Oncol. 26(10), 1626-1634 (2008).

31 Davies H, Bignell GR, Cox C et al. Mutations of the BRAF gene in human cancer. Nature 417(6892), 949-954 (2002).

32 Wan PT, Garnett MJ, Roe SM et al. Cancer Genome Project. Mechanism of activation of the RAF-ERK signaling pathway by oncogenic mutations of B-RAF. Cell 116(6), 855-867 (2004).

33 Yuen ST, Davies H, Chan TL et al. Similarity of the phenotypic patterns associated with BRAF and KRAS mutations in colorectal neoplasia. Cancer Res. 62(22), 6451-6455 (2002).

34 Manceau G, Imbeaud S, Thiébaut R et al. Hsa-miR-31-3p expression is linked to progression-free survival in patients with KRAS wild-type metastatic colorectal cancer treated with anti-EGFR therapy. Clin. Cancer Res. 20(12), 3338-3347 (2014).

35 Engelman JA, Luo J, Cantley LC. The evolution of phosphatidylinositol 3-kinases as regulators of growth and metabolism. Nat. Rev. Genet. 7(8), 606-619 (2006).

36 Samuels Y, Wang Z, Bardelli A et al. High frequency of mutations of the PIK3CA gene in human cancers. Science 304(5670), 554 (2004).

37 Abubaker J, Bavi P, Al-Harbi S et al. Clinicopathological analysis of colorectal cancers with PIK3CA mutations in Middle Eastern population. Oncogene 27(25), 3539-3545 (2008).

38 De Roock W, De Vriendt V, Normanno N, Ciardiello F, Tejpar S. KRAS, BRAF, PIK3CA, and PTEN mutations: implications for targeted therapies in metastatic colorectal cancer. Lancet Oncol. 12(6), 594-603 (2011).

39 Yin Y, Shen WH. PTEN: a new guardian of the genome. Oncogene 27(41), 5443-2453 (2008).

40 Pandolfi PP. P-TEN exciting years: from the cytosol to the nucleus and back to keep cancer at bay. Oncogene 27, 5386 (2008).

41 Martin V, Landi L, Molinari F et al. HER2 gene copy number status may influence clinical efficacy to anti-EGFR monoclonal antibodies in metastatic colorectal cancer patients. Br. .. Cancer 108(3), 668-675 (2013).

42 Laurent-Puig P, Cayre A, Manceau G et al. Analysis of PTEN, BRAF, and EGFR status in determining benefit from cetuximab therapy in wild-type KRAS metastatic colon cancer. J. Clin. Oncol. 27(35), 5924-5930 (2009). 
43 Sartore-Bianchi A, Di Nicolantonio F, Nichelatti M et al. Multi-determinants analysis of molecular alterations for predicting clinical benefit to EGFR-targeted monoclonal antibodies in colorectal cancer. PLoS ONE4(10), e7287 (2009).

44 Brand TM, Iida M, Wheeler DL. Molecular mechanisms of resistance to the EGFR monoclonal antibody cetuximab. Cancer Biol. Ther: 11, 777-792 (2011).

45 Viloria-Petit A, Crombet A, Jothy $S$ et al. Acquired resistance to the antitumor effect of epidermal growth factor receptorblocking antibodies in vivo: a role for altered tumor angiogenesis. Cancer Res. 61, 5090-5101 (2001).

46 Ciardiello F, Bianco R, Caputo R et al. Antitumor activity of ZD6474, a vascular endothelial growth factor receptor tyrosine kinase inhibitor, in human cancer cells with acquired resistance to antiepidermal growth factor receptor therapy. Clin. Cancer Res. 10, 784-793 (2004).

47 Diaz LA Jr, Williams RT, Wu J et al. The molecular evolution of acquired resistance to targeted EGFR blockade in colorectal cancers. Nature 486(7404), 537-534 (2012).

48 Misale S, Yaeger R, Hobor $\mathrm{S}$ et al. Emergence of KRAS mutations and acquired resistance to anti-EGFR therapy in colorectal cancer. Nature 486(7404), 532-536 (2012).

- Observed the pre-existence of a small percentage of cells with these genetic alterations, but also the de novo acquisition of a $K R A S$ mutation under the pressure of cetuximab treatment.

49 Misale S, Arena S, Lamba $S$ et al. Blockade of EGFR and MEK Intercepts heterogeneous mechanisms of acquired resistance to anti-EGFR therapies in colorectal cancer. Sci. Transl. Med. 6(224), $224 \mathrm{ra} 26$ (2014).

- Resistant cells consistently displayed MEK and ERK activation, which persisted after EGFR blockade.

50 Bardelli A, Corso S, Bertotti A et al. Amplification of the MET receptor drives resistance to anti-EGFR therapies in colorectal cancer. Cancer Discov. 3(6), 658-673 (2013).

51 Wheeler DL et al. Epidermal growth factor receptor cooperates with Src family kinases in acquired resistance to cetuximab. Cancer Biol. Ther: 8, 696-703 (2009).

$52 \mathrm{Lu}$ Y, Li X, Liang $\mathrm{K}$ et al. Epidermal growth factor receptor (EGFR) ubiquitination as a mechanism of acquired resistance escaping treatment by the anti-EGFR monoclonal antibody cetuximab. Cancer Res. 67(17), 8240-8247 (2007).

53 Montagut C, Dalmases A, Bellosillo B et al. Identification of a mutation in the extracellular domain of the epidermal growth factor receptor conferring cetuximab resistance in colorectal cancer. Nat. Med. 18(2), 221-223 (2012).

-. S492R EGFR mutation conferred resistance to cetuximab maintaining susceptibility of tumor cells to panitumumab.

54 Tougeron D, Cortes U, Ferru A et al. Epidermal growth factor receptor $(E G F R)$ and KRAS mutations during chemotherapy plus anti-EGFR monoclonal antibody treatment in metastatic colorectal cancer. Cancer Chemother: Pharmacol. 72(2), 397-403 (2013).

55 Yonesaka K, Zejnullahu K, Okamoto I et al. Activation of ERBB2 signaling causes resistance to the EGFR-directed therapeutic antibody cetuximab. Sci. Transl. Med. 3(99), 99ra86 (2011).
56 Yang X, Zhang X, Mortenson ED, Radkevich-Brown O, Wang Y, Fu YX. Cetuximab mediated tumor regression depends on innate and adaptive immune responses. Mol. Ther: 21, 91-100 (2012).

57 Garrido G, Rabasa A, Garrido C et al. Preclinical modeling of EGFR-specific antibody resistance: oncogenic and immuneassociated escape mechanisms. Oncogene 33(24), 3129-3139 (2013).

58 Baldus SE, Schaefer KL, Engers R, Hartleb D, Stoecklein NH, Gabbert HE. Prevalence and heterogeneity of KRAS, BRAF, and PIK3CA mutations in primary colorectal adenocarcinomas and their corresponding metastases. Clin. Cancer Res. 16, 790-799 (2010).

59 Bouchahda M, Karaboué A, Saffroy R et al. Acquired KRAS mutations during progression of colorectal cancer metastases: possible implications for therapy and prognosis. Cancer Chemother: Pharmacol. 66, 605-609 (2010).

60 Lefebure B, Charbonnier F, Di Fiore F et al. Prognostic value of circulating mutant DNA in unresectable metastatic colorectal cancer. Ann. Surg. 251(2), 275-280 (2009).

-• Observed that the presence of circulating mutant DNA was predictive of clinical outcome.

61 Bazan V, Bruno L, Augello C et al. Molecular detection of TP53, Ki-Ras and p16INK4A promoter methylation in plasma of patients with colorectal cancer and its association with prognosis. Results of a 3-year GOIM (Gruppo Oncologico dell'Italia Meridionale) prospective study. Ann. Oncol. 17(Suppl. 7), 84-90 (2006).

62 Van Cutsem E, Kohne CH, Hitre E et al. Cetuximab and chemotherapy as initial treatment for metastatic colorectal cancer. N. Engl. J. Med. 360, 1408-1417 (2009).

63 Van Cutsem E, Köhne CH, Láng I et al. Cetuximab plus irinotecan, fluorouracil, and leucovorin as first-line treatment for metastatic colorectal cancer: updated analysis of overall survival according to tumor KRAS and BRAFmutation status. J. Clin. Oncol. 29(15), 2011-2019 (2011).

64 Ciardiello F, Lenz H-J, Kohne C-H et al. Treatment outcome according to tumor RAS mutation status in CRYSTAL study patients with metastatic colorectal cancer (mCRC) randomized to FOLFIRI with/without cetuximab. Presented at: 2014 ASCO Annual Meeting. Chicago, IL, USA, 30 May-3 June 2014.

65 Maughan TS, Adams RA, Smith GS et al. Addition of cetuximab to oxaliplatin-based first-line combination chemotherapy for treatment of advanced colorectal cancer: results of the randomised Phase 3 MRC COIN trial. Lancet 377, 2103-2114 (2011).

66 Tveit KM, Guren T, Glimelius B et al. Phase III trial of cetuximab with continuous or intermittent fluorouracil, leucovorin, and oxaliplatin (Nordic FLOX) versus FLOX alone in first-line treatment of metastatic colorectal cancer: the NORDIC-VII study. J. Clin. Oncol. 30(15), 1755-1762 (2012).

67 Heinemann V, von Weikersthal LF, Decker T et al. Randomized comparison of FOLFIRI plus cetuximab versus FOLFIRI plus bevacizumab as first-line treatment of KRAS wild-type metastatic colorectal cancer: German AIO study KRK-0306 (FIRE-3). Presented at: 2013 ASCO Annual Meeting. Chicago, IL, USA, 31 May-4 June 2013. 
68 Jonker DJ, O'Callaghan CJ, Karapetis CS et al. Cetuximab for the treatment of colorectal cancer. N. Engl. J. Med. 357(20), 2040-2048 (2007).

69 De Roock W, Jonker DJ, Di Nicolantonio F et al. Association of KRAS p.G13D mutation with outcome in patients with chemotherapy-refractory metastatic colorectal cancer treated with cetuximab. JAMA304(16), 1812-1820 (2010).

70 Bokemeyer C, Bondarenko I, Hartmann JT et al. Efficacy according to biomarker status of cetuximab plus FOLFOX-4 as first-line treatment for metastatic colorectal cancer: the OPUS study. Ann. Oncol. 22 (7), 1535-1546 (2011).

71 Freeman DJ, Ogbagabriel S, Xie J et al. Mechanisms mediating the negative interaction between oxaliplatin $(\mathrm{Ox})$ and epidermal growth factor receptor (EGFR) inhibitors in patients (pts) with $K R A S$ mutant (MT) colorectal cancer (CRC). Presented at: ASCO Annual Meeting 2012. Chicago, IL, USA, 1-5 June 2012.

72 Kim JC, Kim SY, Cho DH et al. Novel chemosensitive single-nucleotide polymorphism markers to targeted regimens in metastatic colorectal cancer. Clin. Cancer. Res. 17(5), 1200-1209 (2011).

73 Gebhardt F, Zanker KS, Brandt B. Modulation of epidermal growth factor receptor gene transcription by a polymorphic dinucleotide repeat in intron 1. J. Biol. Chem. 274(19), 13176-13180 (1992).

74 Zhang W, Gordon M, Press OA et al. Cyclin D1 and epidermal growth factor polymorphisms associated with survival in patients with advanced colorectal cancer treated with cetuximab. Pharmacogenet. Genomics 16(7), 475-483 (2006).

75 Graziano F, Ruzzo A, Loupakis F et al. Pharmacogenetic profiling for cetuximab plus irinotecan therapy in patients with refractory advanced colorectal cancer. J. Clin. Oncol. 26(9), 1427-1434 (2008).

76 Lurje G, Nagashima F, Zhang W et al. Polymorphisms in cyclooxygenase- 2 and epidermal growth factor receptor are associated with progression-free survival independent of K-ras in metastatic colorectal cancer patients treated with single-agent cetuximab. Clin. Cancer Res. 14(23), 7884-7895 (2008).

77 Pander J, Gelderblom H, Antonini NF et al. Correlation of FCGR3A and EGFR germline polymorphisms with the efficacy of cetuximab in KRAS wild-type metastatic colorectal cancer. Eur. J. Cancer46(10), 1829-1834 (2010).

- EGFR intron 1 long variant was significantly related to a decreased progression-free survival in the cetuximab arm.

78 Loupakis F, Antoniotti C, Cremolini C et al. Prospective study of EGFRintron 1 (CA)n repeats variants as predictors of benefit from cetuximab and irinotecan in chemorefractory metastatic colorectal cancer (mCRC) patients. Pharmacogenomics /. 14(4), 322-327 (2014).

79 Zhang W, Gordon M, Schultheis AM et al. FCGR2A and FCGR3A polymorphisms associated with clinical outcome of epidermal growth factor receptor expressing metastatic colorectal cancer patients treated with single-agent cetuximab. J. Clin. Oncol. 20(25), 3712-3718 (2007).

80 Bibeau F, Lopez-Crapez E, Di Fiore F et al. Impact of Fc $\{$ gamma\}RIIa-Fc $\{$ gamma\}RIIIa polymorphisms and
$K R A S$ mutations on the clinical outcome of patients with metastatic colorectal cancer treated with cetuximab plus irinotecan. J. Clin. Oncol. 1(27), 1122-1129 (2009).

81 Geva RBVJ, Fountzilas G, Yoshino T et al. An international consortium study in chemorefractory metastatic colorectal cancer (mCRC) patients (pts) to assess the impact of FCGR polymorphisms on cetuximab efficacy. J. Clin. Oncol. 29(Suppl.) Abstract 3528 (2011).

82 Zhang W, Winder T, Ning Y et al. A let-7 microRNAbinding site (polymorphism in 3'-untranslated region of $K R A S$ gene predicts response in wild-type KRAS patients with metastatic colorectal cancer treated with cetuximab monotherapy. Ann. Oncol. 22(1), 104-109 (2011).

83 Graziano F, Canestrari E, Loupakis F et al. Genetic modulation of the Let-7 microRNA binding to KRAS 3 '-untranslated region and survival of meta-static colorectal cancer patients treated with salvage cetuximab-irinotecan. Pharmacogenomicsf. 10(5), 458-464 (2010).

84 Parmar S, Schumann C, Rüdiger S et al. Pharmacogenetic predictors for EGFR-inhibitor-associated skin toxicity. Pharmacogenomics f. 13(2), 181-188 (2013).

85 Song X, Zhao Z, Barber B et al. Cost of illness in patients with metastatic colorectal cancer. /. Med. Econ. 14, 1-9 (2011).

86 Fojo T, Grady C. How much is life worth: cetuximab, non-small cell lung cancer, and the $\$ 440$ billion question. J. Natl Cancer Inst. 101, 1044-1048 (2009).

87 Lange A, Prenzler A, Frank M et al. A systematic review of cost-effectiveness of monoclonal antibodies for metastatic colorectal cancer. Eur. J. Cancer 50, 40-49 (2014).

88 Shiroiwa T, Motoo Y, Tsutani K. Cost-effectiveness analysis of KRAS testing and cetuximab as last-line therapy for colorectal cancer. Mol. Diagn. Ther: 14, 375-384 (2010).

89 Mittmann N, Au H, Tu D et al. Prospective cost-effectiveness analysis of cetuximab in metastatic colorectal cancer: evaluation of National Cancer Institute of Canada Clinical trials Group CO.17 trial. J. Natl Cancer Inst. 101, 1182-1192 (2009).

90 Vijayaraghavan A, Efrusy MB, Goke B etal. Cost-effectiveness of KRAS testing in metastatic colorectal cancer patients in the United States and Germany. Int. J. Cancer: 131, 438-445 (2012).

91 Medical Advisory Secretariat. KRAS testing for anti-EGFR therapy in advanced colorectal cancer: an evidence-based and economic analysis. Ont. Health Technol. Assess. Ser: 10, 1-49 (2010).

92 Asseburg C, Frank M, Kohne C et al. Cost-effectiveness of targeted therapy with cetuximab in patients with $K$-ras wild-type colorectal cancer presenting with initially unresectable metastases limited to the liver in a German setting. Clin. Ther: 33, 482-497 (2011).

93 Königsberg R, Hulla W, Klimpfinger M et al. Clinical and economic aspects of KRAS mutational status as predictor for epidermal growth factor receptor inhibitor therapy in metastatic colorectal cancer patients. Oncology 81, 359-364 (2011). 
Reproduced with permission of the copyright owner. Further reproduction prohibited without permission. 